\title{
Urgences
}

\section{Les êtres et les choses déposés là}

\section{Madeleine Gagnon}

Numéro 30, décembre 1990

L'autre du texte

URI : https://id.erudit.org/iderudit/025623ar

DOI : https://doi.org/10.7202/025623ar

Aller au sommaire du numéro

Éditeur(s)

Urgences

ISSN

0226-9554 (imprimé)

1927-3924 (numérique)

Découvrir la revue

Citer ce document

Gagnon, M. (1990). Les êtres et les choses déposés là. Urgences, (30), 31-36. https://doi.org/10.7202/025623ar d'utilisation que vous pouvez consulter en ligne.

https://apropos.erudit.org/fr/usagers/politique-dutilisation/ 


\section{Les êtres et les choses déposés là 1}

\section{Madeleine Gagnon}

C'est par le silence qu'on entre dans la peau des mots. Quand on passe cette porte (ou qu'on emprunte cette voie, ce chemin - peut-être bien un petit sentier), on habite une demeure toute spéciale, à la fois proche et chérie, lointaine et inquiétante, elle est multiple et remplie de mots, de phrases pour nommer le monde (les êtres et les choses), elle se dit et se chante de mille et une façons, mais elle n'a qu'un seul nom qui annonce tous les mots dérobés au grand livre de la vie: elle s'appelle poésie.

C'est par le silence qu'on entre dans la demeure de la poésie.

Autrement, on n'entendrait pas les poèmes qu'elle contient.

Autrement, on serait sourds. Et muets. Et aveugles, car tous les sens sont reliés. C'est l'affaire de la poésie de dire ces liens. On serait sans goût, presque plus de saveurs, ni d'odeurs et comment toucher, comment goûter le toucher de la peau quand on ne hume plus? C'est tout lié.

Je crois bien que l'ultime visée de la psychanalyse - je ne parle pas de toutes les entreprises sectaires qui malheureusement s'affublent de ce nom -, son issue la plus vraie et la plus heureuse (guérir, n'est-ce pas d'abord et tout le temps mieux connaitre le bonheur?), pour tout sujet prêt à s'engager dans un long voyage sans commencement ni fin, mais non sans fondement, la leçon la plus chère qu'on peut en dégager concerne ce que je nommerais simplement l'intelligence du silence, celle des seuls mots qui surgissent de lui, sans que la signification n'en soit nécessairement révélée, mais qui viennent, à la fois incertains et assurés, dire à celui ou à celle qui parle, sous la forme murmurée du signe rêvé, le lieu fluctuant et le temps fuyant où se dessine, à travers l'autre, la rencontre de soi.

C'est par la porte du silence de l'autre que la parole, même inaudible, ose inventer les mots du retour à soi.

1 Premier chapitre d'un livre en préparation, à paraitre sous le titre Ecriture et psychanalyse. 
32

Pour le sujet de parole, c'est le silence de l'autre qui donne accès à son rêve. « Rêver l'Autre ", écrit René Major, annonf̧ant le registre dans lequel se déploie sa pensée, immensément complexe et nuancée - enfin! et pour cause -, du transfert ${ }^{2}$.

L'écriture poétique et la psychanalyse n'ont pas pour objet la création de quelque chose qui fera mieux comprendre la beauté ou encore donnera une meilleure connaissance de l'inconscient. Elles n'ont pas de but esthétique ou scientifique. Elles laissent volontiers ces projets aux savants, esthéticiens ou théoriciens de la chose littéraire ou de l'inconscient. Elles sont des pratiques (des arts?) et les sujets qui s'y adonnent n'apprendront qu'à jouer du silence, comme par exemple on joue du piano, à jouer avec les mots dégagés du silence de l'autre comme les notes le sont de nulle part, du moins on dirait.

Si j'ai toujours écrit (des poèmes, des proses), c'est que j'ai toujours aimé rêver l'autre, tout-autre, être ou chose.

Si j'ai toujours écrit, c'est que les mots du silence de l'autre, dans l'acte même de l'écriture, avaient ce don (étrange, merveilleux) de me parler mieux. Disons que je les entends mieux dans l'écriture que partout ailleurs. Quelle que soit la forme dans laquelle se coulera cette écriture (poèmes, proses). Ces mots, on les entsnd aussi dans l'amour, quand l'amour, au-delà d'un nécessaire amoncellement de mots, devient ce tremblement de terre corporel et redonne enfin au corps son initiale opalescence.

Et si, toute jeune ('avais 22 ans), je fus attirée, par de flous méandres et un très mince fil de connaissances théoriques, je fus embarquée dans cette longue aventure que fut (et encore est) la psychanalyse, c'est que j'avais pressenti, en quelque sorte, cette autre vaste demeure du silence dans laquelle le rêve de l'autre (être, chose) peut se vivre - quasi intégralement - et rencontrer là le rêve des mots entrevus dans la demeure de la poésie.

2 René Major, Rêver l'Autre, Paris, Aubier-Montaigne. Et aussi L'agonie du jour, chez le mème éditeur. En ces livres, ó combien difficiles, se trouvent les propos les plus lumineux qu'il m'ait été donné de lire autour de la pratique du transfert (non pas du concept - René Major indique par ailleurs, et à travers toutes sortes de voies, les limites conceptuelles de ce terme); propos qui osent sortir des ornières scientifiques classiques de la théorie psychanalytique (et de toutes les théories psy, de façon générale) et qui ouvrent à une pensée du poétique sur laquelle j'aimerais revenir dans un chapitre ultérieur. 
À travers le tumulte et la cacophonie du monde, il est souvent dit: parlez plus fort, je ne vous entends pas, mais jamais: écoutez mieux ce qui se parle tout bas. Quand le brouillard opaque entoure la terre, il n'est pas d'injonction à mieux regarder, mais seulement une voix intérieure qui s'allume, invitant l'œil à descendre vers elle pour mieux voir êtres et choses déposés au dedans.

Là, proche et lointain, familier mais non moins étranger, se trouve le poème du monde.

Il est enfoui sous le chemin oblique de la «troisième oreille». Il n'advient qu'après un formidable séisme de tout le continent corporel, après une «luxation * définitive du « tympan ", un tremblement de l'être entier, un vacillement même du logos, comme l'écrit Jacques Derrida ${ }^{3}$ et, par lui, le logos devient littéralement loxos (luxere: luxation, fracture, effraction).

Le poème est cette effraction de l'être dans le brouillard du rêve de l'autre (être, chose) et dans la nuit des temps. Comme la parole de la séance (la parole pleine), il n'a ni commencement ni fin (il est interminable), il s'écrit brisé, fracturé. Il est ce fragment ravi au silence éternel des dieux et permet cet envol outre-frontières vers une contrée sans limites où tous les poètes du monde ont aligné ces notes de l'autre-rêvé qui flottent au hasard du silence des ans (du silex) et qui se captent au toucher de l'écoute incertaine.

Du poème, René Char écrit dans "Le poème pulvérisé «: "Il m'apprit à voler au-dessus de la nuit des mots, loin de l'hébétude des poèmes à l'ancre * 4 .

"Hébétude des poèmes à l'ancre "que l'encre et le sang, et le lait et les larmes, et toutes les grandes eaux du monde contiennent et noient quand est franchi pour de bon - mais c'est toujours à recommencer - le seuil de la demeure de la nuit des mots.

Ainsi l'écrivait déjà le poète biblique: «Ma bouche énonce la sagesse, et le murmure de mon cœur l'intelligence. Je tends l'oreille à quelque proverbe, je résous sur la lyre mon énigme " (Psaume 48).

3 Jacques Derrida, Eperons ou les styles de Nietzsche, coll. «Champs", Paris, Flammarion.

4 René Char, CEuvres complètes, coll. «Bibliothèque de la Pléiade ", Paris, Gallimard, p. 261. 
34

Pour cette demeure du silence de l'autre, la clef de l'énigme est le rêve et l'énigme se résout dans le chant.

Résoudre l'énigme, de soi et de l'autre, sur la voix percussive qui seule atteint la peau des mots, sa poussée, sa pulsion, mais aussi sur la voix pensive, car si la demeure du poème n'est pas à proprement parler philosophique - le logos subit l'ébranlement du loxos ${ }^{5}$, elle n'empêche toutefois pas de penser. La poésie, c'est la pensée qui chante. Et qui vibre comme un être vivant.

C'est aussi la pensée qui prie, qui invoque et implore, mais ses incantations sont dirigées vers l'absence, adressées nulle part et vers nulle patrie, car, là où elle porte, les dieux se sont depuis longtemps (toujours?) éclipsés.

La poésie, c'est l'éternelle orpheline des dieux évanouis. Mais, paradoxalement, la demeure où s'énonce poème ou parole pleine permet, à celle et celui qui osent déambuler dans ses chambres et couloirs habités de fantômes attentifs, la sortie du vaste orphelinat terrestre.

Les fantômes attendent encore - et attendront sans cesse et toujours - des mots les animant à nouveau et qui feront s'ouvrir leurs bouches éteintes.

is Chaque sujet vivant chérit ses fantômes comme autant de trésors secrets; intransmissibles; chaque sujet vivant les retrouve parfois au détour d'une pensée esquissée, d'une phrase rêvée, d'un bris de mot livrant sous sa coque des lettres greffées qui, de très loin et depuis longtemps - mais collées au plus près du corps opaque -, racontent des bribes d'histoires fossilisées. Ces lettres, la psychanalyse les nomme pulsions ${ }^{6}$. Et les poètes, depuis toujours, n'ont pas eu besoin des termes théoriques de la psychanalyse pour faire leur miel de cet insolite destin des choses et des êtres échoués, tels des revenants, dans des formules innommées que les mots du poème feront advenir au langage. Les mots de la parole pleine.

5 J'emplöie librement ces termes derridiens, sans guillemets: ils me conviennent et me convient à l'écriture.

6 Je pense ici au " legs de Freud ", à ses héritiers très studieux et je me réfère plus précisément à certains textes: "L'instance de la lettre dans l'inconscient" de Jacques Lacan (Ecrits I); Psychanalyser de Serge Leclaire et, surtout, La carte postak de Jacques Derrida. Je n'entre pas dans les détails subtils des débats, tel n'est pas mon propos. Je traverse cet horizon à vol d'oiseau, délibérément. 
Ce langage, on le sait, est tout autre que celui de la norme. Là où gisent les revenants du corps opaque (des êtres, des choses), il n'y a pas de lexique structuré en grammaires et syntaxes. La seule rhétorique qui puisse les ressusciter, le temps d'une phrase ou d'un verset ou même d'un murmure, est celle des profondeurs dont Baudelaire sut si bien nous entretenir, longtemps avant que la psychanalyse put prétendre à certaines assises scientifiques, lettres de noblesse dont les poètes, presque toujours maudits, se sont, tant bien que mal, passés.

À la petite école, longtemps avant que le terme «inconscient * me fut connu et bien avant aussi la lecture des poètes, j'avais en moi créé un monde où rêves de nuit et chants du poème habitaient le même pays. Par des détours, qui pourtant échappaient à mon intelligence raisonnable, je les avais fait s'épouser. J'acceptais comme allant de soi le mystère de leur rencontre et savais, comme à travers les mots qui me manquaient, que jamais les mots appris ne sauraient expliquer leur mutuelle adhésion.

C'est dans la pratique de l'écoute flottante qu'il m'était donné d'assister au cérémonial répétitif de leur mariage.

Souvent, pendant les analyses grammaticales et syntaxiques, les règles sues, tout en gardant une oreille aux bribes d'explications utiles, je dérivais vers la contrée de ces épousailles, là où, sur les cimes ou dans les abîmes, on entend le chant des mots.

Toujours, le chant venait quand j'avais marché sur deux petits chemins parallèles qui, soudain, se rencontraient: celui, toujours déroutant, de mes rêves nocturnes et l'autre, non moins rempli d'insolites détails, de toutes ces rêveries issues de certains mots adorés, accrochés à eux, agglutinés même - comme si certains mots, sous leur gangue terreuse, avaient le don de posséder plusieurs noyaux que je croyais célestes.

J'avais le sentiment que le sentier du rêve, quand il touchait enfin celui de la rêverie des mots, conjuguait, mieux que tout autre, les seules phrases qui me permettraient d'entrer dans les chapitres du grand livre éternel de la vie. Je savais devoir m'y avancer sur la pointe du cœur - et je m'y appliquais; chaque chapitre étant perçu (entendu) comme une maison offerte au seul passant discret (et distrait). Et je m'y engageais, de temps en temps, assurée que la traversée du 
36

livre me donnerait un jour le secret, irrévélé aux indiscrets faiseurs de phrases, de la plus énigmatique chose: l'éternité.

Je pensais qu'un jour, après l'une des cérémonies de noces, s'ouvrirait un chemin menant tout droit à l'éternité - c'était toujours en mon esprit, de l'autre côté de la mer, entendue à cette époque comme la-fin-du-monde -; un chemin enfin libre où, avec tous mes alliés venus des rêves et rêveries, je serais ravie, par le cœur de la lettre, dans l'âme des mots.

Je pensais que l'âme des mots se trouvait sous leur peau.

Et je le pense encore.

J'intuitionnais parfois qu'il me faudrait un jour voyager sous ma propre peau pour y suivre à la trace les lettres greffées. Et puis, innocente, je m'éclipsais de cette pensée.

Dans celle-ci, innommable à l'époque, je me sentais devenir complètement nébuleuse. Je ramenais alors tout mon être dans la classe, m'absorbant à l'étude, pratiquant mes lettres comme on fait ses gammes, remettant à plus tard - mais "plus tard* alors n'était jamais trop loin - mon désir du grand livre où l'âme des choses coulerait sans contraintes dans le cceur des mots.

Après, à la plus grande école, je sus que pour apprendre une langue on lit les écrivains, mes rêveries de vocables se démultiplièrent et, comme j'entrais aussi dans l'âge du fantasme, la promesse du livre se fit à la fois plus prégnante et lointaine.

Tout me parut alors si complexe et les chemins d'accès si touffus, si mêlés, que j'en restai studieusement à la lecture des poètes, aux explications de textes, aux récitations et dissertations. Tout en faisant régulièrement encore mes gammes, j'avais atteint le stade de l'interprétation. Pour un temps, je perdis l'usage de ma troisième oreille. Adieu, écoute flottante! Il me fallait ètrer dans les couloirs froidement infinis du logos.

C'est par «l'imagination transcendentale» de Kant (enseigné par Paul Ricœur) que je pus entrevoir enfin la sortie des voies arides. Savais vingt et un ans. C'était l'âge de la majorité.

Toutes ces années-là d'apprentissage sévère, je gardais, enfoui en moi, comme une promesse secrète, le grand désir du livre où s'uniraient pour toujours, sous la peau des mots, les êtres et les choses rêvés.

Ici, dans ce livre, j'écris le destin de ce vœu. 